\title{
Soluble Fc $\gamma$ Receptor III in Human Plasma Originates from Release by Neutrophils
}

Tom W. J. Huizinga," Masja de Haas," Marion Kleijer," Jan H. Nuijens,“ Dirk Roos," and Albert E. G. Kr. von dem Borne** *Central Laboratory of the Netherlands Red Cross Blood Transfusion Service and Laboratory for Experimental and Clinical Immunology, University of Amsterdam, Amsterdam; and ${ }^{\ddagger}$ Department of Haematology,

Academic Medical Center, Amsterdam, The Netherlands

\begin{abstract}
FcRIII (the CD16-antigen), a low affinity receptor for IgG, is expressed by neutrophils, natural killer lymphocytes, and macrophages. We have developed a sensitive radioimmunoassay to quantify FcRIII. A soluble form of FcRIII was identified in human plasma. Immunoprecipitation of FcRIII from plasma showed that the plasma form of FcRIII has an identical electrophoretic mobility as the FcRIII expressed by neutrophils. Moreover, the plasma form of FCRIII exhibited the same polymorphism as does the neutrophil FcRIII. The neutrophil expresses the phosphatidylinositol-linked form of FCRIII, encoded by the gene FCRIII-1. Because it is not known whether this gene is also active in nonhematopoietic cells, we analyzed patients with an acquired clonal disorder of their hematopoietic cells, paroxysmal nocturnal hemoglobinuria (PNH). PNH patients appeared to have a strongly reduced expression of FcRIII on their neutrophils. The concentration of FcRIII in the plasma of these patients was also reduced, indicating that plasma FcRIII originates from neutrophils. A patient deficient in FCRIII-1 but with a normal expression of FCRIII-2 had no soluble FcRIII in her plasma, also indicating that plasma FcRIII originates from neutrophils.

The electrophoretic mobility of the protein backbone of plasma FcRIII and FcRIII released by activated neutrophils was identical, whereas deglycosylated FcRIII obtained from a lysate of neutrophils migrated slower. This indicates that plasma FcRIII originates from activation-induced release by neutrophils. Stimulation of neutrophils or neutrophil cytoplasts (closed membrane vesicles filled with cytoplasm) with low concentrations of FMLP $\left(10^{-9}-10^{-8} \mathrm{M}\right)$ or phorbol myristate acetate $(1-10 \mathrm{ng} / \mathrm{ml})$ induced a dose-dependent release of FcRIII. The plasma concentration of FcRIII was relatively constant (range $\mathbf{4 0 - 2 8 0 \%}$ of the mean). Soluble FcRIII was also detected in inflamed joint fluids of arthritis patients, suggesting that FCRIII is also released by activated neutrophils in vivo. (J. Clin. Invest. 1990. 86:416-423.) Key words: Fcr receptor III • neutrophils $\bullet$ NA polymorphism
\end{abstract}

\section{Introduction}

The neutrophil is the most abundant cell of the cellular defence system against microorganisms. This cell can be induced

Address correspondence and reprint requests to Dr. A. E. G. Kr. von dem Borne, c/o Publication Secretariat, Central Laboratory of The Netherlands Red Cross Blood Transfusion Service, P. O. Box 9406, 1006 AK Amsterdam, The Netherlands.

Received for publication 21 September 1989 and in revised form 26 February 1990.

J. Clin. Invest.

(C) The American Society for Clinical Investigation, Inc.

0021-9738/90/08/0416/08 $\$ 2.00$

Volume 86, August 1990, 416-423 by inflammatory signals to adhere to the endothelium and to move to the inflammatory focus, where the neutrophil degranulates and produces toxic oxygen metabolites. The neutrophil membrane is adapted to fulfill these various functions. Adhesiveness to the endothelium and chemotaxis is induced by bacterial cell-wall-derived peptides, such as FMLP, or complement fragments, such as C5a. These compounds induce an enhanced expression of adhesion receptors (for a review, see 1). The respiratory burst, degranulation, and phagocytosis are supposed to be mediated mainly by Fc $\gamma$ receptors and complement receptors (2).

Human Fc $\gamma$ receptor III (FcRIII) ${ }^{1}$ or CD16-antigen is expressed on neutrophils, natural killer (NK) lymphocytes, and macrophages. Two genes are coding for this receptor, FcRIII-1 and FcRIII-2 $(3,4)$. The FcRIII-1 mRNA encodes a protein with a short (four amino acids) cytoplasmic domain, whereas the FcRIII-2 mRNA encodes a protein with a cytoplasmic domain of 25 amino acids. The FcRIII- 1 protein is proteolytically cleaved during post-translational processing and coupled to a phosphatidylinositol (PI) anchor, whereas the FcRIII-2 protein appears to be a transmembrane protein. The PI linkage of neutrophil FcRIII was confirmed by the virtual absence of FcRIII on neutrophils from patients with paroxysmal nocturnal hemoglobinuria (PNH) an acquired clinical disorder affecting PI tail attachment $(5,6)$. Neutrophils appear to express only the PI-linked form of FcRIII, whereas NK lymphocytes and macrophages only express the transmembrane form of FcRIII (3).

Apparently, glycosylation of neutrophil FcRIII (FcRIII-1) differs from that of NK FcRIII (FcRIII-2). Neutrophil FcRIII has a slower electrophoretic mobility in SDS-PAGE than NK FcRIII, and deglycosylated neutrophil FcRIII migrates faster than NK FcRIII (7). Moreover, the neutrophil FcRIII shows a genetically determined heterogeneity, the neutrophil antigen (NA) system NA1NA2 (8-10). This polymorphism is caused by differences in a few amino acids between the NA1 form of FcRIII-1 and the NA2 form of FcRIII-1. Two changes (Asn to Ser on position 63 and Asp to Asn on position 82 ) result in two extra glycosylation sites in the NA2 form $(3,11,12)$. Mainly due to this glycosylation difference, the NA polymorphism leads to a different electrophoretic mobility of FcRIII from NA1-homozygous, NA1NA2-heterozygous, and NA2-homozygous individuals.

In this report we describe the effect of various stimulating

1. Abbreviations used in this paper: CGD, chronic granulomatous disease; DFP, diisopropylfluorophosphate; FcRIII, Fc $\gamma$ receptor III; FcRIII-1, the gene encoding the phosphoinositol-linked form of Fc $\gamma$ receptor III; FCRIII-2, the gene encoding the transmembrane form of Fc $\gamma$ receptor III; GPI-PLC, glycosylphosphatidylinositol-specific phospholipase C; GPI-PLD, GPI-phospholipase D; NA, neutrophil antigen; NK, natural killer; PNH, paroxysmal nocturnal hemoglobinuria; PI, phosphatidylinositol. 
compounds on neutrophil FcRIII expression as well as on the release of FcRIII. Moreover, we analyzed the mechanism of the activation-induced release of this receptor. To study whether such a release also occurs in vivo, we measured the occurrence of soluble FcRIII in plasma as well as in joint fluids. Because it has been reported that neutrophil FcRIII (5) and NK FcRIII (13) are released upon activation, we analyzed the cellular origin of soluble FcRIII in plasma.

\section{Methods}

$M A b$. MAb against FcRIII, CLBFcRgran1, BW209/2, and 3G8 have been described $(7,8)$. MAb against the NA1 allotype of neutrophil FcRIII, CLBgran11, has also been described $(7,8)$. BW209/2 was a generous gift of Dr. R. Kurle (Behring Werke AG, Marburg, FRG). A cross-blocking study showed that these MAb are directed against different epitopes of FcRIII. MAb B13.9 is directed against a PI-linked activation antigen of $100 \mathrm{kD}$ (CD67-antigen) and has been described in detail $(14,15)$. MAb 15D9 was used as CD45 MAb and was a generous gift of Dr. R. van Lier (CLB).

Cell separation. Blood from volunteers or patients was anticoagulated with trisodium citrate. Mononuclear leukocytes and platelets were removed by centrifugation over Percoll (Pharmacia Fine Chemicals AB, Uppsala, Sweden) with a specific gravity of $1.076 \mathrm{~g} / \mathrm{ml}$. Erythrocytes were subsequently lysed with isotonic $\mathrm{NH}_{4} \mathrm{Cl}$ at $4^{\circ} \mathrm{C}$. The remaining cells were $>95 \%$ neutrophils.

Cytoplasts from neutrophils were prepared as previously described (16). Briefly, neutrophils were suspended in $12.5 \%$ (wt/vol) Ficoll-70 containing $10 \mu \mathrm{g}$ of cytochalasin B per $\mathrm{ml}$. This suspension was layered on a discontinuous gradient of 16 and $25 \%$ (wt/vol) Ficoll-70. Cytochalasin B $(10 \mu \mathrm{g} / \mathrm{ml})$ was present throughout the gradient. After centrifugation $\left(30 \mathrm{~min}, 81,000 \mathrm{~g}, 34^{\circ} \mathrm{C}\right)$, the cytoplasts were harvested from the interface of the 12.5 and $16 \%$ Ficoll solutions. The cytoplasts were washed two times and resuspended in incubation medium. The volume of a cytoplast is about one-third the volume of an intact cell, as deduced from the content of lactate dehydrogenase (cytoplasm) and alkaline phosphatase (plasma membrane). The amount of lysosomal enzymes of the cytoplasts was $<5 \%$ compared to intact cells, as judged by the lysozyme content.

Sandwich RIA. CLBFcRgran 1 was coupled to $\mathrm{CNBr}$-activated Sepharose $4 B(10 \mathrm{mg}$ of protein to $1 \mathrm{~g}$ of Sepharose) and diluted to $0.1 \%$ (wt/vol) in PBS (10 mM sodium phosphate, $140 \mathrm{mM} \mathrm{NaCl}, \mathrm{pH}$ 7.4) containing EDTA $(10 \mathrm{mM})$ and Tween $(0.1 \%$, wt/vol). $500 \mu \mathrm{l}$ of this Sepharose suspension were incubated with the supernatant or lysate of $10^{6}$ activated neutrophils or with a specified amount of human plasma, for $5 \mathrm{~h}$ at room temperature by head-over-head rotation in 2-ml polystyrene tubes. The Sepharose beads were washed five times with $1.5 \mathrm{ml}$ of saline and incubated for $16 \mathrm{~h}$ at room temperature with saturating concentrations of ${ }^{125} \mathrm{I}$-labeled BW209/2. Sample dilutions of BW209/2 were made in PBS containing EDTA (10 mM), Tween-20 (0.1\%, wt/vol), and 5\% (vol/vol) normal goat serum. Subsequently, the beads were washed five times with saline and the bound radioactivity was counted in a multi-gamma counter (LKB Instruments, Inc., Gaithersburg, MD).

Immunoprecipitation from plasma. This was performed with a modification of the method described by Ory et al. (10). CLBFcRgran 1 was covalently coupled to Sepharose beads $(10 \mathrm{mg}$ of protein to $1 \mathrm{~g}$ of Sepharose). The beads were diluted to $10 \%$ (wt/vol) in PBS. $500 \mu \mathrm{l}$ of the beads were incubated with $1 \mathrm{ml}$ of plasma. The beads were washed five times in saline buffered with $10 \mathrm{mM}$ phosphate. Subsequently, the beads were layered on a discontinuous $10-20 \%$ sucrose gradient and spun down. After another washing step with saline buffered with 10 $\mathrm{mM}$ Tris, $\mathrm{pH} 6.8$, FcRIII was eluted with $1.0 \mathrm{M}$ glycine, $\mathrm{pH} 2.5$, and neutralized with $1.0 \mathrm{M}$ Tris, $\mathrm{pH} 8$. The eluted material was extensively dialyzed against PBS and labeled with $3.7 \mathrm{MBq}{ }^{125}$ Iodine in the Iodogen method. This material was extensively dialyzed against PBS to remove all free ${ }^{125}$ iodine, and aliquots of it were treated with either $3 \mathrm{G} 8$
MAb (anti-FcRIII), CLBFcRgran 1 MAb, or CD45 MAb and analyzed on SDS-PAGE. The lanes in which the 3G8 and CLBFcRgran 1-precipitate were analyzed showed the characteristic FcRIII-smear, whereas the lane in which CD45 precipitate was analyzed was empty.

Immunoprecipitation from cells. Neutrophils $\left(2 \times 10^{7}\right)$, preincubated with $1 \mathrm{mM}$ DFP, were labeled with $37 \mathrm{MBq}{ }^{125}$ iodide by the Iodogen method according to the manufacturer's instructions (Pierce Chemical Co., Rockford, IL). After labeling, the cells were suspended in saline buffered with $10 \mathrm{mM}$ phosphate $(\mathrm{pH} \mathrm{7.4)}$ and supplemented with $\mathrm{CaCl}_{2}(0.6 \mathrm{mM}), \mathrm{MgCl}_{2}(1.0 \mathrm{mM})$, glucose $(1 \mathrm{mg} / \mathrm{ml})$, and human albumin $(0.5 \% \mathrm{wt} / \mathrm{vol})$. The cells were treated as stated below under Neutrophil treatment. Subsequently, the cells were lysed in $1 \%(\mathrm{wt} / \mathrm{vol})$ NP-40 in the presence of the protease inhibitors EDTA (5 mM), PMSF $(2 \mathrm{mM})$, and soybean-trypsin inhibitor $(200 \mathrm{ng} / \mathrm{ml})$. Immunoprecipitation from the medium or lysate of labeled cells with CLBFcRgran 1 covalently coupled to Sepharose was carried out as described previously $(5,9)$. Immunoprecipitated proteins were subjected to SDSPAGE and autoradiography. $N$-glycanase treatment was performed according to the manufacturer's instructions (Genzyme, Boston, MA). Briefly, FcRIII immunoprecipitated from either the lysate or the medium of $2 \times 10^{6}$ neutrophils was incubated for $18 \mathrm{~h}$ with $10 \mathrm{U}$ of $N$-glycanase per $\mathrm{ml}$ at $37^{\circ} \mathrm{C}$, pH 8.6.

Neutrophil treatment. Radiolabeled neutrophils were stimulated for $10 \mathrm{~min}$ at $37^{\circ} \mathrm{C}$ with FMLP and were spun down to separate medium from cells. The medium was centrifuged for $30 \mathrm{~min}$ at 12,000 $g$ to remove possible neutrophil membrane vesicles. Centrifugation for $60 \mathrm{~min}$ at $100,000 \mathrm{~g}$ yielded the same results (not shown). After glycosylphosphatidylinositol-specific phospholipase C (GPI-PLC) digestion, the cells were spun down and the medium was centrifuged afterwards for $30 \mathrm{~min}$ at $12,000 \mathrm{~g}$. To determine the sensitivity of the release of FcRIII for serine protease inhibitors, neutrophils $\left(3 \times 10^{6} / \mathrm{ml}\right)$ or neutrophil cytoplasts $\left(18 \times 10^{6} / \mathrm{ml}\right)$ were stimulated with phorbol myristate acetate (PMA) $(100 \mathrm{ng} / \mathrm{ml})$ for $10 \mathrm{~min}$ in the presence of various protease inhibitors. The neutrophils or neutrophil cytoplasts were spun down to separate medium from cells. The medium was then centrifuged for $30 \mathrm{~min}$ at $12,000 \mathrm{~g}$ before its FcRIII content was measured in the sandwich RIA. The effectiveness of diisoprophyl fluorophosphate (DFP) and PMSF was tested simultaneously in a chromogenic assay, based on the inhibition of elastase-mediated degradation of $N$-methoxysuccinyl-ala-ala-pro-val-p-nitroanilide (Sigma Chemical Co., St. Louis, MO).

Patients. Venous blood was obtained from healthy employees in our institute and from various categories of patients. This blood was anticoagulated by EDTA and spun down at $1,700 \mathrm{~g}$ for $6 \mathrm{~min}$. Subsequently, the plasma was harvested and spun down at $12,000 \mathrm{~g}$ for 30 min. Plasma from chronic granulomatous disease (CGD) patients was provided by Dr. R. S. Weening. The patients were characterized by an inability of their neutrophils to produce superoxide. Plasma from PNH patients, characterized by an absence of PI-linked proteins on neutrophils (CD16, CD24, and CD67) as well as by a positive Acid Ham test and a positive sucrose lysis test, was provided by Dr. C. E. van der Schoot. Plasma from septic shock patients was provided by Drs. L. G. Thijs, C. E. Hack, and J. H. Nuijens. These patients were characterized by a temperature $>38.5^{\circ} \mathrm{C}$, a blood pressure $<100 / 60$, a heart rate $>100$ beats per min, and a positive bacterial blood culture. Plasma from arthritic patients was provided by J. J. Abbink, C. E. Hack and A. J. G. Swaak. These patients were investigated for a swollen and red knee and were diagnosed as indicated in the legend to Fig. 6.

Plasma from patients with idiopathic neutropenia was provided by Drs. W. H. Ouwehand and R. Kuijpers. These patients were characterized by a peripheral granulocyte count of $<1.5 \times 10^{9} /$ liter. Plasma from patients with Wegener's granulomatosus was provided by Dr. K. M. Dolman and Dr. R. Goldschmeding. These patients were characterized by the presence of anti-neutrophil-cytoplasmic antibodies and clinical symptoms (fever, malaise, weight loss, and biopsy-proven granulomatous inflammation and/or vasculitis in the upper respiratory tract or kidney). Patients with active systemic lupus erythematosus (SLE) were characterized by the presence of anti-DNA antibodies 
in the Farr assay; plasma samples of these patients were provided by Dr. R. Smeenk and Dr. A. J. G. Swaak.

\section{Results}

FcRIII in human plasma. A catching RIA to quantify FcRIII was developed. Fig. 1 shows that our sandwich RIA detected FcRIII in human plasma. The specificity of the RIA to detect FcRIII in plasma was checked by addition of the supernatant of GPI-PLC-treated neutrophils to plasma. This increased the FcRIII concentration as measured by the RIA. The specificity of the RIA was further tested by several negative controls. No positive signal was obtained when CD45 MAb coupled to Sepharose beads were used instead of anti-FcRIII MAb (CLB FcRgran1) coupled to Sepharose beads. When we used goat serum instead of human plasma or when we added ${ }^{125}$ I-labeled CD45 MAbs instead of ${ }^{125}$ I-labeled BW209/2 (anti-FcRIII), the results were also negative. The specificity of the assay was also confirmed by preclearing samples of human plasma with an IgG-Sepharose column. This decreased the FcRIII concentration as measured by the RIA. It also showed that the plasma form of FcRIII still bound IgG.

To determine soluble FcRIII in plasma, we used pooled plasma from 38 healthy volunteers as a reference. The range of FcRIII concentrations of each individual who contributed to the pool was $40-280 \%$ (see also Fig. 6); $100 \%$ being the concentration of FcRIII in the pooled plasma. Assuming that one BW209/2 MAb binds to one FcRIII molecule, this would account for a plasma concentration of 1.3-12 nM, with $5 \mathrm{nM}$ as the mean. The range of the FcRIII concentrations in the plasma of 25 randomly chosen patients admitted to a general hospital was 2-9 $\mathrm{nM}$. The mean concentration of their plasma FcRIII was also $5 \mathrm{nM}$. No correlation $(r=0.22)$ between the amount of plasma FcRIII and the number of circulating neutrophils was observed $(n=25)$.

Cellular origin of FcRIII. Neutrophil FcRIII shows the NA polymorphism (8-10). This polymorphism causes a different electrophoretic mobility in SDS-PAGE of neutrophil FcRIII derived from NA1NA1, NA1NA2 and, NA2NA2 donors. Therefore, plasma FcRIII was precipitated from plasma obtained from NA1NA1, NA1NA2, or NA2NA2 donors by anti-FcRIII MAb (CLBFcRgran1) coupled to Sepharose. This material was eluted, neutralized, and dialyzed. Subsequently, it was iodinated with ${ }^{125}$ Iodine and analyzed on SDS-PAGE. FcRIII was also precipitated from a lysate of neutrophils

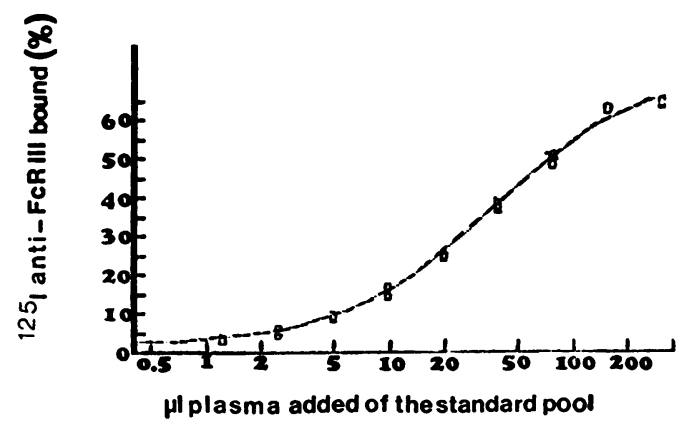

Figure 1. Detection of FcRIII in human plasma. EDTA plasma (320, $160,80,40,20,10,5,2.5$, and $1.25 \mu l)$ was tested in the sandwich RIA as described in Methods. (which express FcRIII-1) and NK cells (which express FcRIII2). Fig. 2 shows that the electrophoretic mobility of plasma FcRIII corresponds with that of neutrophil FcRIII, in its size heterogeneity as well. This indicates that the plasma form of FcRIII originates from neutrophils. To prove this, we precleared plasma from an NAINA1 homozygous donor with a MAb (CLB gran 11) that only reacts with the NA1 allotype of neutrophil FcRIII and not with the NK form of FcRIII. CLBgran 1 1-Sepharose removed all FCRIII from the plasma of an NA1NA1 donor. This provides another indication that plasma FcRIII originates from neutrophils.

Recently, we identified a female patient without neutrophil-FcRIII expression, either membrane bound or cytoplasmic. The neutrophils of this patient had a normal expression of other PI-linked proteins, such as CD24 and CD67, indicating that she did not suffer from PNH. Her NK lymphocytes and cultured monocytes had a normal FcRIII expression (this patient has a genomic deficiency of the FcRIII-1 gene, submitted for publication). This patient had no soluble FcRIII in her plasma (compared to the curve in Fig. 1, she had $<5 \%$ of the normal average content of soluble FcRIII). This confirmed the specificity of our RIA and that soluble FcRIII originate from FcRIII-1.

However, it might be possible that the FcRIII-1 gene is also active in not yet identified nonhematopoietic cells. To investigate this possibility, we analyzed patients with PNH (an ac-

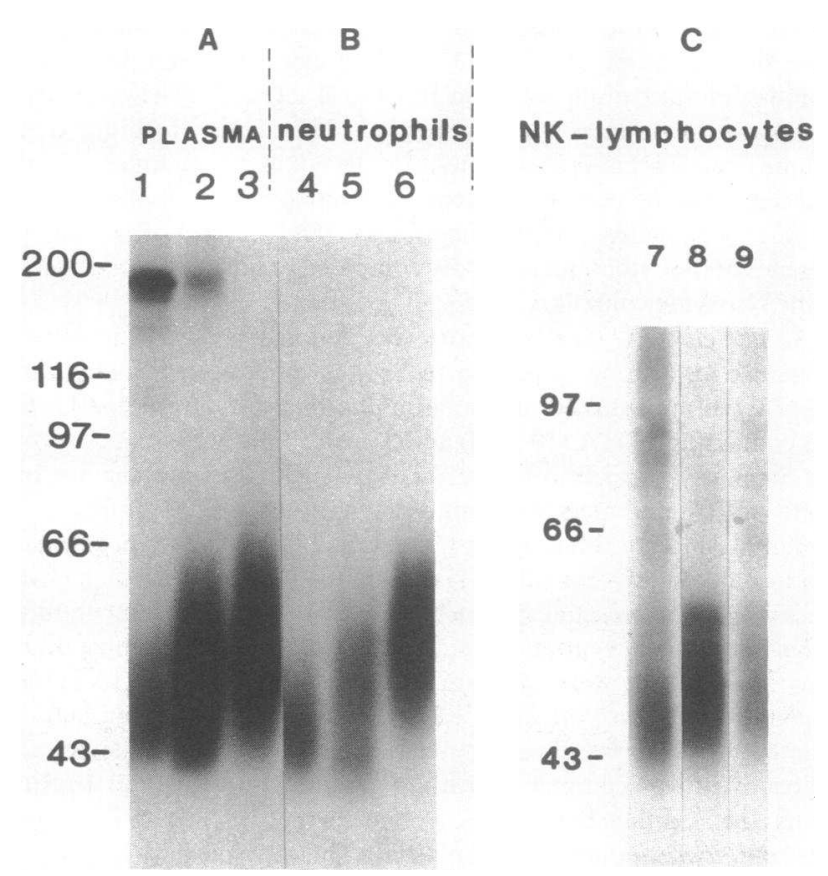

Figure 2. Autoradiograph of SDS-PAGE of FCRIII isolated from either plasma $(A)$, lysate of neutrophils $(B)$ or lysate of lymphocytes $(C)$. Lanes 1,4 , and 7 contain material from an NA1NA1 donor; lanes 2,5 , and 8 from an NA1NA2 donor, and lanes 3,6 , and 9 from an NA2NA2 donor. Size markers are indicated on the left. Note that the $M_{r}$ of plasma FcRIII is identical to that of neutrophil FcRIII and different from NK FcRIII. The band at $150 \mathrm{kD}$, that is seen on lanes 1 and 2 , was removed from the gel in lane 3 . Although only the relevant portion of the autoradiogram is shown, we would like to emphasize that the front of lanes 1,2 , and 3 was thicker and more intense than the front of the other lanes. 
quired clonal disorder of the hematopoietic cells). The neutrophils from patients with PNH have a very low expression of FcRIII $(5,6)$, because these cells cannot express PI-linked proteins. The mean concentration of FcRIII in PNH plasma is $25 \% \pm 5 \%$ (range $12-37 \%$ ) compared to the mean plasma concentration of the standard pool (Fig. 6). This indicates that plasma FcRIII originates from neutrophil FcRIII.

Besides material with the $M_{\mathrm{r}}$ of FcRIII, we also precipitated a protein of $150-160 \mathrm{kD}$ under nonreducing conditions. When this 150-160-kD band was cut out (lane 3 of Fig. 2) and analyzed again under reducing conditions, we found two bands of 50 and $25 \mathrm{kD}$. This suggests that we copurified IgG with FcRIII under these conditions. Moreover, we also found a

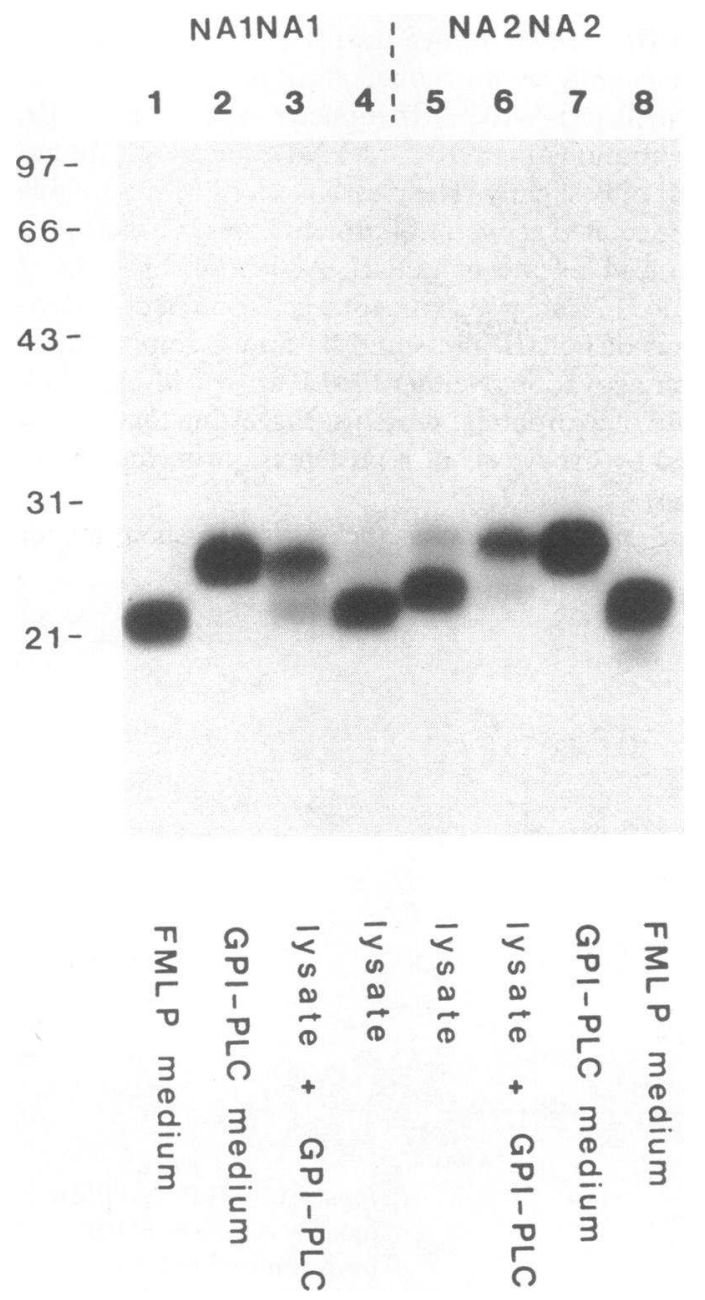

Figure 3. Autoradiograph of SDS-PAGE of FcRIII after either FMLP stimulation or GPI-PLC treatment of ${ }^{125}$ I-labeled neutrophils. After immunoprecipitation, the proteins were digested with $\mathrm{N}$-glycanase to remove $N$-linked sugars. Lanes $1-4$ contain material from an NA1NA1 donor, lanes 5-8 contain material from an NA2NA2 donor. FcRIII was immunoprecipitated by anti-FcRIII MAb from the lysate of unstimulated neutrophils (lanes $3,4,5$, and 6), from the $12,000 \mathrm{~g}$ supernatant of the medium of GPI-PLC-treated neutrophils (lanes 2 and 7), or from the $12,000 \mathrm{~g}$ supernatant of FMLPstimulated neutrophils (lanes 1 and 8 ). The immunoprecipitates from lanes 3 and 6 were digested with GPI-PLC to confirm that the shift in electrophoretic mobility is caused by hydrolysis of the PI anchor. Size markers are indicated on the left.
140-150-kD band after $N$-glycanase treatment (Fig. 4) (an IgG molecule has $2-4 \%$ carbohydrate). We also precipitated plasma FcRIII and blotted it to nitrocellulose. Subsequently, it was incubated with an anti-human IgG conjugate, and with an anti-mouse IgG conjugate. Only the anti-human IgG conjugate yielded a positive reaction. These experiments prove that it was indeed human IgG that was copurified.

Mechanism of release of FcRIII. Neutrophil FcRIII is a PI-linked protein. Therefore, we investigated whether the release of FCRIII was mediated by a phospholipase or a proteinase. If this release is caused by the activation of an endogenous

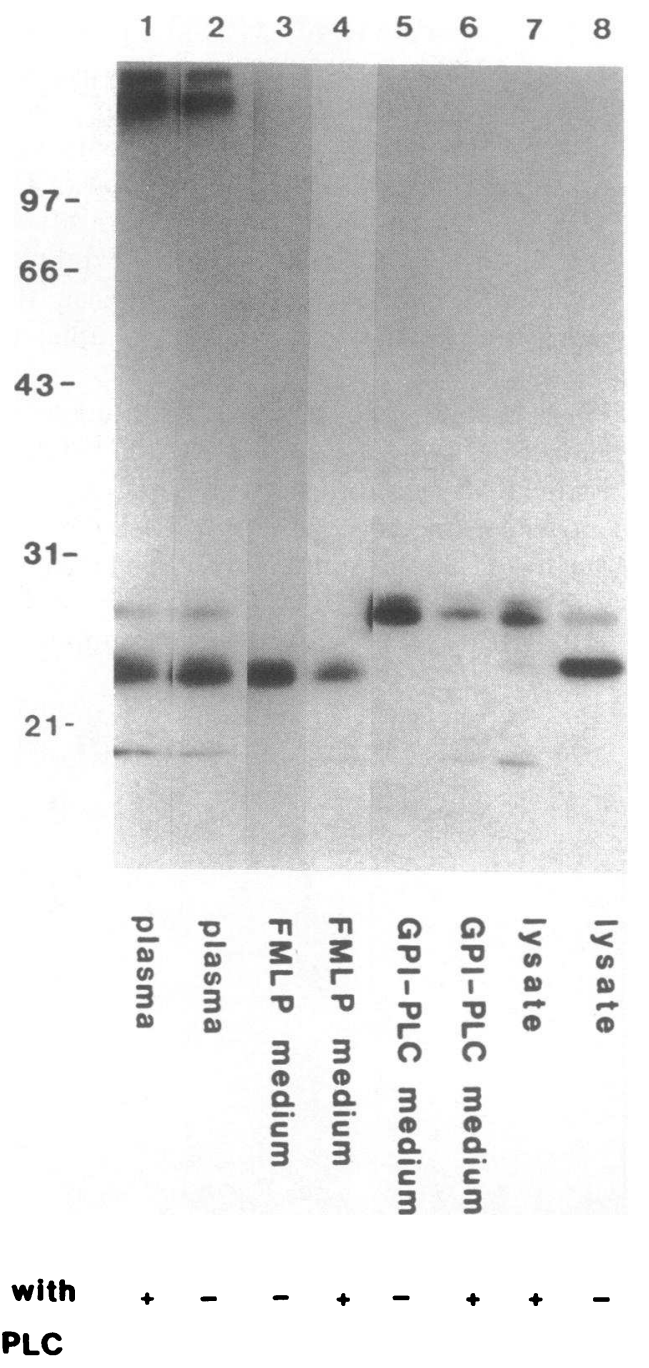

Figure 4. Autoradiograph of SDS-PAGE of deglycosylated FCRIII. The immunoprecipitate was digested by $\mathrm{N}$-glycanase to remove $\mathrm{N}$ linked sugars. All lanes contain material from an NA2NA2 donor. Lanes 1 and 2, FcRIII isolated from plasma; lanes 3 and 4, FcRIII from the $12,000 \mathrm{~g}$ supernatant of FMLP-stimulated neutrophils; lanes 5 and 6, FcRIII from the $12,000 \mathrm{~g}$ supernatant of GPI-PLCtreated neutrophils; lanes 7 and 8 , FcRIII from the lysate of neutrophils. The immunoprecipitates from lanes $1,4,6$, and 7 were digested with GPI-PLC to confirm that there was no shift in electrophoretic mobility in lanes 1,4 , and 6 caused by hydrolysis of the PI anchor as observed in lane 7 . All samples were analyzed on a nonreducing $12 \%$ SDS-PAGE. Note that deglycosylated FCRIII from plasma has the same electrophoretic mobility as the deglycosylated, FMLP-released form of neutrophil FcRIII. 
phospholipase, one would expect other PI-linked proteins to be released as well. However, CD67, a PI-linked protein, could not be precipitated from the supernatant of FMLP-treated neutrophils, although it was precipitated from the supernatant of GPI-PLC-treated neutrophils (15). These experiments suggested that the PI linkage in itself is not a sufficient condition for shedding upon cell activation.

To determine whether FCRIII released by GPI-PLC differed from FcRIII released by FMLP, we compared the relative molecular mass of the deglycosylated proteins. (No differences were observed when the fully glycosylated proteins were analyzed.) Fig. 3 shows that deglycosylated FcRIII from NA1NAl donors has an $M_{\mathrm{r}}$ of 23-24 kD, whereas that of NA2NA2 donors has an $M_{\mathrm{r}}$ of 25-26 kD. Immunoprecipitation from the supernatant of GPI-PLC-digested neutrophils showed that FcRIII released by GPI-PLC migrated slower. This shift in electrophoretic mobility after removal of diacylglycerol was confirmed by GPI-PLC digestion of FcRIII that had been previously immunoprecipitated from a lysate of neutrophils. In contrast, FMLP-released FcRIII exhibited the fastest electrophoretic mobility. This might indicate that FMLP induces release of FCRIII by a proteinase rather than by a phospholipase.

In support of this, we found that the simultaneous incubation of neutrophils with DFP and PMA inhibited the release of FcRIII. Preincubation of the cells with DFP followed by washing had no effect on the release of FcRIII: DFP had to be present during neutrophil stimulation. High concentrations of PMSF ( $2 \mathrm{mM}$ ) or EDTA (5 mM) reduced the PMA-induced release of FcRIII by $60 \%$. Neither phosphoramidon (10 nM), thiorphan (10 $\mathrm{nM})$ (both inhibitors of neutral proteinases), nor soybean-trypsin inhibitor $(10 \mathrm{ng} / \mathrm{ml})$ affected the PMA-induced release of FcRIII. To control the integrity of a signal transduction pathway, we tested whether these proteinase inhibitors had an effect on neutrophil activation as measured by oxygen consumption. Only EDTA ( $5 \mathrm{mM})$ and DFP (5 mM) had a small inhibitory effect on the PMA-induced activation of the respiratory burst. Although these inhibitors might either block the signal transduction pathway that leads to release of FcRIII or the enzyme responsible for release of FcRIII, these inhibition experiments suggest that FcRIII is released by a proteinase. We tested several proteinases (elastase, pronase, and an extract of azurophilic granules) and found that these enzymes release FcRIII. This might suggest that FcRIII is released via exocytosis of granule proteins.

The concentration of stimulating compounds that induce release of FcRIII was determined next. Fig. 5 shows that activation of neutrophils by low concentrations of the bacterial tripeptide FMLP $\left(10^{-9}-10^{-8} \mathrm{M}\right)$ induced release of FCRIII. Higher concentrations than $10^{-8} \mathrm{M}$ FMLP increased the release of FcRIII only slightly. The phorbol ester PMA, a soluble nonreceptor-mediated activator of protein kinase $C$, induced a more pronounced release of FcRIII. Activation by $1 \mathrm{ng}$ of PMA/ml induced 7\% release of the total cell content of FcRIII. The expression of FcRIII (measured by flow cytometry) was decreased after activation. Neither FMLP at $10^{-8} \mathrm{M}$ nor PMA induces release of azurophilic proteins, suggesting that FcRIII is not released by exocytosis of a proteinase from the azurophilic granules.

To exclude granule proteins as the enzymes responsible for
I

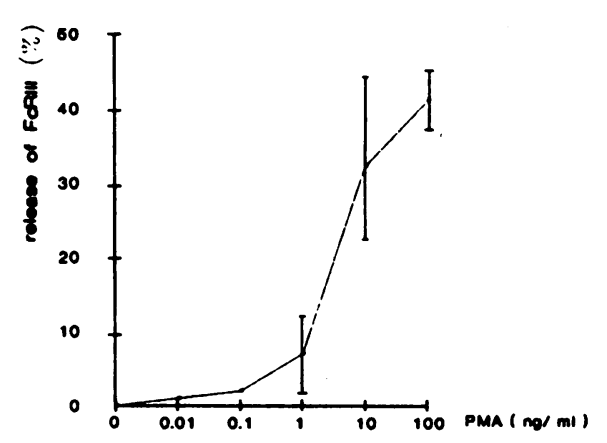

II

A

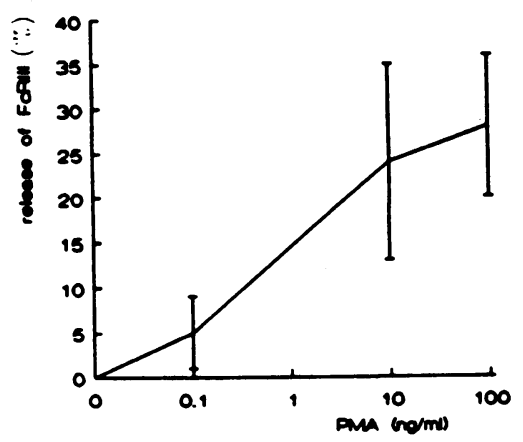

B

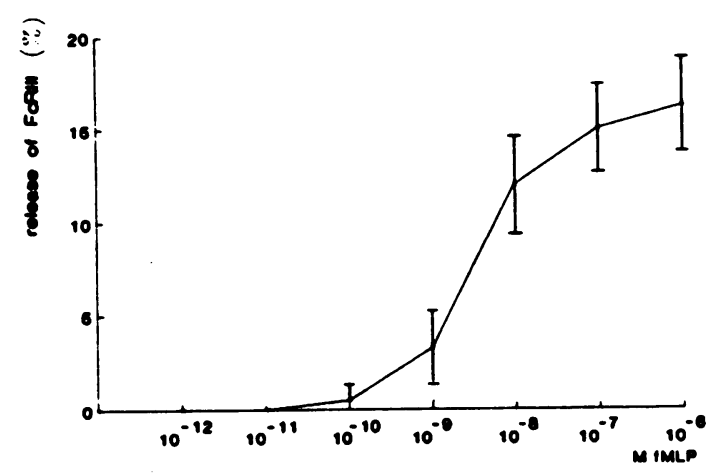

B

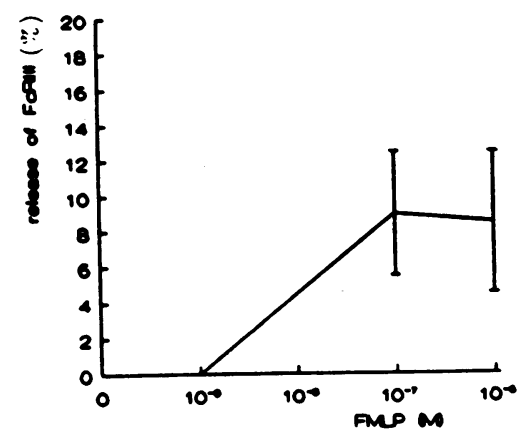

Figure 5. FMLP- and PMAinduced release of FcRIII from neutrophils $(I)$ and from neutrophil cytoplasts (II). The release of FcRIII was measured by a sandwich RIA. Neutrophils and neutrophil cytoplasts were stimulated for $10 \mathrm{~min}$ with various concentrations of FMLP $(A)$ or PMA $(B)$. The percentage release by unstimulated neutrophils, $6 \% \pm 3 \%$ (mean \pm SD, $n=8$ ), or by unstimulated neutrophil cytoplasts, $15 \% \pm 4 \%(n=6)$, was subtracted from values. 
shedding of FcRIII, we made cytoplasts from neutrophils. Cytoplasts are closed membrane vesicles filled with cytoplasm but devoid of nuclei and granules, still capable of executing membrane-bound functions such as the generation of superoxide. As shown in Fig. 5, FMLP and PMA caused a stimulus-induced release of FcRIII from cytoplasts. The FMLP- or PMA-induced release of FcRIII from cytoplasts was also inhibited by DFP and PMSF.

Plasma FcRIII may be identical to FMLP-released neutrophil FcRIII. Plasma contains a high concentration of proteinase inhibitors. Therefore, neutrophils were isolated, resuspended in plasma, and stimulated with PMA. These experiments showed that FcRIII is also released in the presence of plasma. Subsequently, we investigated whether FcRIII in plasma results from activation-induced release by neutrophils. Fig. 4 shows that the plasma form of FcRIII has the same electrophoretic mobility after deglycosylation as the FcRIII released by FMLP-treated neutrophils. Moreover, the absence of the PI anchor in the plasma form of FcRIII was established by GPI-PLC digestion of FcRIII that had previously been immunoprecipitated from plasma (Fig. 4). These results suggest that the plasma form of FcRIII may originate from release by activated neutrophils.

This hypothesis was tested by the analysis of the concentration of soluble FcRIII in inflamed joint fluids and plasma of arthritic patients. Fig. 6 , columns $A$, shows that when individual patients are analyzed, the soluble FcRIII concentration in synovial fluid $(A-p u$.) was in general higher than the concentration in plasma $(A-p l$.$) . However, in 3$ out of 16 cases this

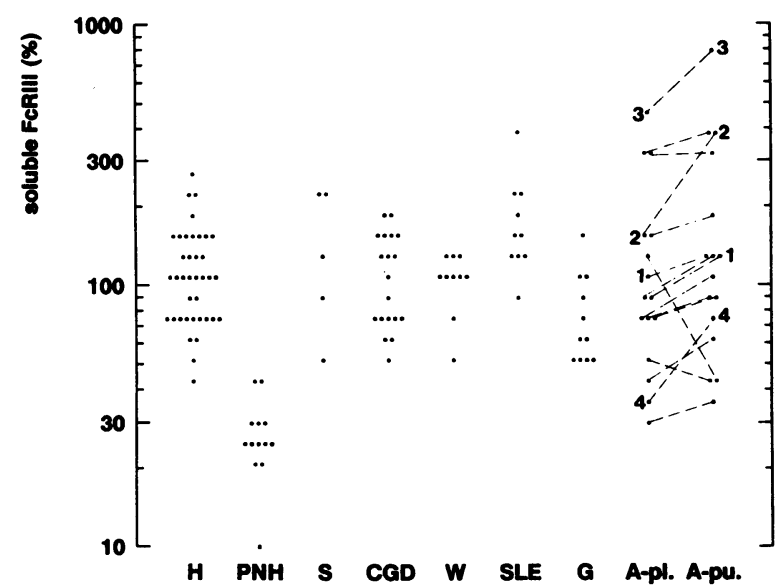

Figure 6. The concentration of FcRIII in plasma from healthy controls or patients. The concentration of FcRIII was compared with a standard plasma pool, consisting of pooled plasma from 38 donors (the concentration in the plasma pool was taken as $100 \%$ ). Each dot represents a different individual. $\mathrm{H}$ indicates healthy controls $(n$ $=38$; $\mathrm{PNH}, n=12$; $\mathrm{S}$ indicates septic shock patients $(n=5)$; $\mathrm{W}$ indicates patients suffering from Wegener's granulomatosis $(n=10)$; CGD, $n=10$; G indicates granulocytopenic patients $(n=10)$; SLE, $n=10 ;$ A indicates arthritic patients $(n=16)$. A-pu. indicates the concentration of FCRIII in the punctate, harvested from an inflamed knee, whereas A-pl. indicates the concentration in plasma. Patients 1 and 2 were both diagnosed as seronegative oligo-arthritis, patient 3 was diagnosed as M. Bechterew, patient 4 was diagnosed with gout, and the other patients were both diagnosed with monoarthritis of unknown origin. Note that the plasma concentration of FcRIII was in general lower than the concentration of FcRIII in synovial fluid. concentration was lower than in plasma. This could be related to the activity of the inflammation rather than to diffusion of FcRIII from plasma. To estimate the diffusion of proteins from plasma to synovial fluid the ratio of synovial albumin versus plasma albumin was determined by Dr. J. J. Abbink. This ratio was always smaller than 0.7 . So, soluble FcRIII seems to be "produced" in the area of inflammation where neutrophils are supposed to be activated.

However, the concentration of FcRIII in plasma from septic-shock patients was within the normal range (see Fig. 6). In these septic-shock patients, the concentration of soluble FcRIII was $138 \% \pm 65 \%$ compared to the standard pool, whereas the elastase and the lactoferrin content were significantly increased [elastase $622 \% \pm 195 \%$ (mean \pm SD) of control values; lactoferrin $406 \% \pm 399 \%$ (mean $\pm S D$ ) of control values]. In patients suffering from chronic granulomatous disease, Wegener's granulomatosis, SLE, or neutropenia, the concentration of soluble FcRIII was within the normal range (Fig. 6), although in SLE patients the concentration of soluble FcRIII tended to be slightly elevated $(164 \% \pm 64 \%)$.

\section{Discussion}

Origin of plasma FcRIII. Our data show that the concentration of soluble FcRIII in plasma is high $(\sim 5 \mathrm{nM})$. The reduced concentration of FcRIII in PNH plasma indicates that soluble FcRIII is produced by a cell originating from one of the hematopoietic cell lineages $(5,6)$. The presence of the neutrophil-specific heterogeneity in the plasma form of FcRIII indicates that plasma FcRIII originates from neutrophils. The reduced concentration of plasma FcRIII in PNH plasma (these patients have a strongly reduced expression of PI-linked FcRIII, but a normal expression of transmembrane FcRIII) and the absence of plasma FcRIII in a patient deficient in FcRIII-1 confirmed that PI-linked FcRIII is the only FcRIII that contributes to the concentration of plasma FcRIII.

Proteolytic release of FcRIII. Our results show that an enzyme, activated by chemotactic concentrations of FMLP or very low concentrations of PMA, causes release of FcRIII. It might be argued that a unique phospholipase is activated that causes specific release of FcRIII and not of other PI-linked proteins, such as the CD67 antigen. However, the decrease in electrophoretic mobility after removing the diacylglycerol by GPI-PLC compared to the FMLP-induced increase in electrophoretic mobility points to a proteolytic release of FcRIII. Moreover, the inhibition of this release by DFP also points to a proteolytic cleavage of FcRIII.

The enzyme responsible for release of FcRIII is presumably membrane bound, because neutrophil cytoplasts also release FcRIII upon stimulation. An alternative explanation might be that the proteinase for FcRIII shedding is located in a compartment that easily fuses with the plasma membrane (e.g., the tertiary granules), thus leading to integration or adherence of this enzyme to the plasma membrane. During preparation of cytoplasts from neutrophils, this degranulation might take place. The dose-dependent activation-induced release of FcRIII from cytoplasts argues against this hypothesis and indicates that the enzyme responsible for release of FcRIII is membrane bound.

DFP must be present during stimulation of neutrophils or cytoplasts to inhibit the activation-induced release of FcRIII. 
This might be due to inhibition of a latent enzyme necessary for FcRIII release that forms a DFP binding site upon cell activation, or to inhibition of the signal transduction pathway that leads to release of FcRIII by DFP. In the latter case a phospholipase may be responsible for FcRIII release, but since all GPI-specific phospholipases described so far (reviewed in 17) have a GPI-PLC or GPI-PLD like activity, we suggest that FcRIII is proteolytically removed from the neutrophil surface.

Because we did not observe a size difference in the fully glycosylated form of FcRIII released by either GPI-PLC or FMLP (9), we suggest that the released form of FcRIII still contains all N-linked oligosaccharides (FcRIII has 4-6 sites available for $\mathrm{N}$-linked glycosylation based on the predicted amino-acid sequence) $(3,18)$. The cleaving of one $\mathrm{N}$-linked oligosaccharide chain is supposed to lead to an enhanced electrophoretic mobility of the parent protein, corresponding with a 2-4-kD reduction in apparent molecular weight (19). Therefore, the substrate sequence of the enzyme responsible for shedding is between amino acid 189 (the most $\mathrm{NH}_{2}$-terminal glycosylation site) and amino acid 206, because the PI anchor attachment is presumed to occur at either Gly (206), Ser (203), Ser (201), Ser (200), Ser (197), or Ala (195) (17, 20, 21).

Cellular implications of the release of FcRIII. Neutrophils can bind IgG-opsonized targets via FcRII and FcRIII. FcRII is involved in activating the respiratory burst, release of granule proteins, and IgG-dependent phagocytosis (22), whereas the sole effector function mediated by FcRIII identified until now is the release of granule proteins (23). The release of FCRIII could result in a more efficient binding of IgG to the receptor (FcRII) that is primarily responsible for the activation of the lytic machinery of the neutrophil. Another possibility might be that contact between a neutrophil and its IgG-opsonized target is terminated by release of FCRIII. We found that FMLP induces release of IgG dimers already bound to neutrophils (unpublished observations). This indicates that FcRIII can be released even after binding of its ligand.

Humoral implications of the release of FCRIII. The normal concentration of FcRIII in the plasma of patients with sepsis, CGD, neutropenia, SLE, or Wegener's granulomatosis indicate that the plasma FcRIII concentration is not simply reflecting the "activational state" of the circulating neutrophils. Because the plasma concentration of FcRIII will depend on the partition coefficient, on the clearance and on the release of FcRIII, this was not expected. Although we have no data on the partition coefficient of soluble FcRIII, it has affinity for IgG. The ratio between the plasma IgG concentration (10 $\mathrm{mg} / \mathrm{ml}$ or $670 \mu \mathrm{M})$ and the concentration of soluble FcRIII (estimated at $5 \mathrm{nM}$ ) is $\sim 100,000 \mathrm{IgG}$ molecules to one FcRIII molecule. Fig. 2 shows that IgG is copurified with plasma FcRIII. This suggests that the plasma form of FcRIII binds to monomeric IgG. This binding will certainly influence the clearance of plasma FcRIII. It should be noted that an FcRIIIIgG complex is apparently precipitated from plasma by an anti-FcRIII MAb (CLBFcRgran 1) that blocked the binding of IgG-opsonized particles to neutrophils $(8,22)$. On the other hand, we have found (unpublished observations) that CLBFcRgran 1 binds to FcRIII on neutrophils after a saturating amount of $\mathrm{IgG}$ complexes has bound to the neutrophils, without removing these complexes. This suggests that some change in FcRIII occurs after binding of IgG.

The function of plasma FcRIII is not clear. It might be that soluble FcRIII is one of the factors that regulate the concen- tration of IgG, either by affecting its production or its turnover. Bich-Thuy et al. (24) have shown that soluble IgG-binding proteins released by neutrophils (presumably FcRIII) selectively suppress the production of IgG by B lymphocytes (for a review, see 25). We found that the plasma concentration of IgG was not correlated to the plasma concentration of FcRIII $(r=0.3, n=36)$. In conclusion, the function of FcRIII in the network regulating the concentration of IgG is not yet understood.

Clinical consequences. The abundant presence of FcRIII on macrophages of the red pulp of the spleen and on the Kupffer cells in the liver prompted Clarkson et al. (26) to investigate whether infusion of (mouse) anti-FcRIII MAb in patients with refractory immune thrombocytopenic purpura blocks the clearance of IgG-opsonized thrombocytes. Initially, this treatment was effective, but both the severe side effects and the high dose of mouse immunoglobulins needed limited this therapeutic application. Because the anti-FcRIII MAb (3G8) that was used in this study also reacts with plasma FcRIII (27), we expect that a large amount of the infused MAb bound to plasma FcRIII. This may have limited the clinical application of this mouse MAb.

The presence of FCRIII (CD16) on CD3-negative, CD4negative, and CD16-positive lymphocytes that are capable of inducing cytotoxicity via FcRIII against NK-resistant targets (28) and its capability of inducing cytokine gene transcription (29), have prompted immunotherapists to produce bispecific MAb that contain one $F(a b)$ region directed against a tumorspecific antigen and one $F(a b)$ region directed against FcRIII. Clinical studies with these bispecific MAb are under way. If plasma FcRIII binds to such bispecific antibodies, this will severely hinder the ability of these reagents to stimulate the contact between an NK lymphocyte and a tumor cell. The in vivo exploitation of the cellular functions mediated by this receptor urges the use of MAb specific for the transmembrane form of FcRIII.

\section{Acknowledgments}

The authors thank Dr. M. Low for his gift of GPI-PLC, Dr. R. Kurle for his gift of BW209/2, Dr. A. van den Ende for providing plasma and data of patients admitted to a general hospital, Drs. R. S. Weening, L. G. Thijs, C. E. Hack, J. J. Abbink, A. J. G. Swaak, C. E. van der Schoot, W. H. Ouwehand, R. Kuijpers, R. Goldschmeding, and K. M. Dolman for providing plasma and data of patients. Moreover, we acknowledge the careful observation of Ms. B. Bossers, which enabled the identification of the FcRIII-deficient patient. Dr. A. Sonnenberg is acknowledged for helpful discussions.

This study was supported by grant 900-512-076 from the Netherlands Foundation for Medical Research, which is subsidized by the Netherlands Organization for Scientific Research.

\section{References}

1. Kuypers, T. W., and D. Roos. 1989. Leukocyte membrane adhesion proteins LFA-1, CR3 and p150,95: a review of functional and regulatory aspects. Res. Immunol. 140:461-486.

2. Borregaard, N. 1988. The human neutrophil: function and dysfunction. Eur. J. Haematol. 41:401-413.

3. Ravetch, J., and B. Perussia. 1989. Alternative membrane forms of FcRIII (CD16) on human NK cells and neutrophils. J. Exp. Med. 170:481-497.

4. Scallon, B. J., E. Scigliano, V. H. Freedman, M. C. Miedel, Y. C. E. Pan, J. C. Unkeless, and J. P. Kochen. 1989. A human 
immunoglobulin $\mathrm{G}$ receptor exists in both polypeptide anchored and phosphatidylinositol-glycan-anchored forms. Proc. Natl. Acad. Sci. USA. 86:5079-5083.

5. Huizinga, T. W. J., C. E. van der Schoot, C. Jost, R. Klaassen, M. Kleijer, A. E. G. Kr. von dem Borne, D. Roos, and P. A. T. Tetteroo. 1988. The PI-linked receptor FcRIII is released on stimulation of neutrophils. Nature (Lond.). 333:667-669.

6. Selvaraj, P., W. F. Rosse, R. Silber, and T. A. Springer. 1988. The major Fc receptor in blood has a phosphatidylinositol anchor and is deficient in paroxysmal nocturnal haemoglobinuria. Nature (Lond.). 333:565-567.

7. Huizinga, T. W. J., M. Kleijer, D. Roos, and A. E. G. Kr. von dem Borne. 1989. Differences between FcRIII of human neutrophils and human $\mathrm{K} / \mathrm{NK}$ lymphocytes in relation to the NA antigen system. In Leucocyte Typing IV. W. Knapp, B. Dörken, W. R. Gilks, E. P. Rieber, R. E. Schmidt, H. Stein, and A. E. G. Kr. von dem Borne, editors. Oxford University Press, Oxford, UK. 582-585.

8. Werner, G., A. E. G. Kr. von dem Borne, M. J. E. Bos, J. F. Tromp, C. M. van der Plas-van Dalen, F. J. Visser, C. P. Engelfriet, and P. A. T. Tetteroo. 1985. Localization of the human NA1 alloantigen on neutrophil Fc $\gamma$ receptors. In Leucocyte Typing II. E. L. Reinherz, B. F. Haynes, L. M. Nadler, and I. D. Bernstein, editors. Springer-Verlag New York Inc., New York. 109-121.

9. Huizinga, T. W. J., M. Kleijer, P. A. T. Tetteroo, D. Roos, and A. E. G. Kr. von dem Borne. 1989. The biallelic neutrophil NA-antigen system is associated with a polymorphism in phospho-inositollinked Fc receptor III (CD16). Blood. 75:213-217.

10. Ory, P. A., I. M. Goldstein, E. E. Kwoh, and S. B. Clarkson. 1989. Characterization of polymorphic forms of Fc receptor III on human neutrophils. J. Clin. Invest. 83:1676-1681.

11. Ory, P. A., N. R. Clark, E. E. Kwoh, S. B. Clarkson, and I. M. Goldstein. 1989. Sequences of complementary DNAs that encode the NA1 and NA2 forms of Fc receptor III on human neutrophils. J. Clin. Invest. 84:1688-1691.

12. Trounstine, M. L., G. A. Peltz, H. Yssel, T. W. J. Huizinga, A. E. G. Kr. von dem Borne, H. Spits, and K. W. Moore. 1990. Reactivity of cloned, expressed human FcRIII isoforms with monoclonal antibodies which distinguish cell type-specific and allelic forms of Fc $\gamma$ RIII. Intern. Immunology. 2:303-310.

13. Lanier, L. L., J. H. Phillips, and R. Testi. 1989. Membrane anchoring and spontaneous release of CD16 (FcRIII) by natural killer cells and granulocytes. Eur. J. Immunol. 19:775-778.

14. Tetteroo, P. A. T., M. J. E. Bos, F. J. Visser, and A. E. G. Kr. von dem Borne. 1986. Neutrophil activation detected by monoclonal antibodies. J. Immunol. 136:3427-3432.

15. Van der Schoot, C. E., T. W. J. Huizinga, S. Gadd, O. Majdic, R. Wijmans, W. Knapp, and A. E. G. Kr. von dem Borne. 1989. Identification of 3 novel PI-linked proteins on granulocytes. In Leucocyte Typing IV. W. Knapp, B. Dörken, W. R. Gilks, E. P. Rieber, R. E.
Schmidt, H. Stein, and A. E. G. Kr. von dem Borne, editors. Oxford University Press, Oxford, UK. 887-891.

16. Roos, D., A. A. Voetman, and L. J. Meerhof. 1983. Functional activities of enucleated human polymorphonuclear leukocytes. J. Cell. Biol. 97:368-377.

17. Low, M. G. 1989. The glycosyl-phosphatidylinositol anchor of membrane proteins. Biochim. Biophys. Acta. 988:427-454.

18. Peltz, G., H. Grundy, R. Lebo, H. Yssel, G. Barsh, and K. W. Moore. 1989. Human FcRIII: cloning, expression, and identification of the chromosomal locus of two receptors for IgG. Proc. Natl. Acad. Sci. USA. 86:1013-1017.

19. Bartles, J. R., L. T. Braiterman, and A. L. Hubbard. 1985. Biochemical characterization of domain-specific glycoproteins of the rat hepatocyte plasma membrane. J. Biol. Chem. 260:12792-12802.

20. Kurosaki, T., and J. V. Ravetch. 1989. A single aminoacid in the glycosyl phosphatidylinositol attachment domain determines the membrane topology of FcRIII. Nature (Lond.). 342:805-807.

21. Lanier, L. L., S. Cwirla, G. Yu, R. Testi, and J. H. Phillips. 1989. Membrane anchoring of a human IgG Fc receptor (CD16) determined by a single amino acid. Science (Wash. DC). 246:1611-1613.

22. Huizinga, T. W. J., F. van Kemenade, L. Koenderman, K. M. Dolman, A. E. G. Kr. von dem Borne, P. A. T. Tetteroo, and D. Roos. 1989. The 40-kD Fc receptor (FcRII) on human neutrophils is essential for the IgG-induced respiratory burst and IgG-induced phagocytosis. J. Immunol. 142:2365-2369.

23. Huizinga, T. W. J., K. M. Dolman, N. J. van der Linden, M. Kleijer, J. H. Nuijens, A. E. G. Kr. von dem Borne, and D. Roos. 1990. PI-linked FcRIII mediates exocytosis of neutrophil granule proteins, but does not mediate initiation of the respiratory burst. J. Immunol. 144:1432-1437.

24. Le Thi Bich-Thuy, C. Samarut, J. Brochier, and J. P. Revillard. 1981. Suppression of the late stages of mitogen-induced human B-cell differentiation by $\mathrm{Fc} \gamma$ receptors released from polymorphonuclear neutrophils. J. Immunol. 127:1299-1303.

25. Daëron, M., and W. H. Fridman. 1985. Fc receptors as regulatory molecules. Ann. Inst. Pasteur Immunol. 136c:383-437.

26. Clarkson, S. B., J. B. Bussel, R. P. Kimberly, J. Valinsky, R. L. Nachman, and J. C. Unkeless. 1986. Treatment of refractory immune thrombocytopenic purpura with an anti-Fc-receptor antibody. $N$. Engl. J. Med. 314:1236-1239.

27. Khayat, D., C. Geffrier, S. Yoon, E. Scigliano, C. Soubrano, M. Weil, J. C. Unkeless, and C. Jacquillat. 1987. J. Immunol. Methods. 100:235-241.

28. Werfel, T., P. Uciechowski, P. A. T. Tetteroo, R. Kurrle, H. Deicher, and R. E. Schmidt. 1989. Activation of cloned natural killer cells via FcRIII. J. Immunol. 142:1 102-1106.

29. Anegon, I., M. C. Cuturi, G. Trinchieri, and B. Perussia. 1988. Interaction of $\mathrm{Fc}$ receptor $(\mathrm{CD} 16)$ with ligand induces transcription of IL-2 (CD25) and lymphokine genes. J. Exp. Med. 167:452-472. 